\title{
VALORACIÓN DEL ESTADO COGNITIVO EN EL ADULTO MAYOR: ÁREAS MÁS AFECTADAS
}

\section{ASSESSMENT OF COGNITIVE \\ STATUS IN THE ELDERLY: \\ MOST AFFECTED AREAS \\ Recibido 11 de marzo 2015 Aceptado 14 de marzo 2015}

Correspondencia:

Sharon Jaqueline Rayón Rayón La Mora Núm. 30, Col. La Loma 56644

Chalco Edo. Méx. shatika_v30@hotmail.com

\section{Autores:}

Sharon Jaqueline Rayón Rayón

Est. Lic. Enfermería ENEO-UNAM

Tania Juárez Romero

Est. Lic. Enfermería ENEO-UNAM

María Cristina Muggenburg Rodriguez Vigil Maestría en Salud Pública

Profesora de Carrea de la ENEO- UNAM

Palabras clave: Estado cognitivo, adulto mayor, deterioro cognitivo.

Key Words: cognitive state, elderly, cognitive défícit. 


\section{RESUMEN}

Introducción: La alteración del estado cognitivo vuelve al adulto mayor dependiente, limita su autocuidado y genera cuidados a largo plazo.

Objetivo: Valorar el estado cognitivo del adulto mayor, identificar las capacidades cognitivas más afectadas según género, edad y escolaridad.

Metodología: Se realizó un estudio descriptivo transversal no probabilístico con enfoque cuantitativo en una casa de rehabililtación y reposo en México D.F. La población fue de 78 adultos mayores de 60 años (41 mujeres y 37 hombres). Se valoró el estado cognitivo con ayuda del (Mini-Mental) cuyo objetivo es establecer un diagnóstico presuntivo de las funciones cognitivas. Se utilizaron cuatro variables: estado cognitivo, género, edad y escolaridad.

Resultados: De las 78 personas el 47.4\% fueron hombres y el $52.6 \%$ mujeres, el $40.5 \%$ tienen de 60 a 69 años, el 32.7\% obtuvo un título universitario, el 23.7\% de la población mostro deterioro severo, el 44.9\% deterioro leve. Las áreas más afectadas fueron: abstracción (cálculo) con 46.2\% de deterioro severo, memoria a corto plazo con 47.4\% de deterioro severo y lenguaje con $17.5 \%$ de deterioro.

Conclusiones: Evaluar y detectar alteraciones cognitivas permitirá concientizar a la familia, buscar modelos de atención y cuidado fortaleciendo el bienestar del adulto mayor.

Palabras clave: Estado cognitivo, adulto mayor, deterioro cognitivo

\section{ABSTRACT}

Introduction: Altered mental state becomes the elderly dependent, limited self-care and generates long-term care.

Objective: To assess the cognitive status of the elderly and identify the cognitive abilities most affected by gender, age and education.

Methodology: A cross-sectional study of non probabilistic descriptive quantitative approach was conducted in a rehabilitation and rest house in Mexico DF. The sample was 78 adults over 60 years, 47 women and 37 men. We made an assessment of cognitive status using the Minimental which objetive to establish a diagnosis of cognitive functions. Were considered four variables: Cognitive status, gender, age and education.

Results: Of the 78 people found that $47.4 \%$ were male and $52.6 \%$ female, $40.5 \%$ was found in the group of $60-69$ years, $32.1 \%$ of seniors earned a university de gree in $23.7 \%$ of the population severe deterioration was observed, 44.9\% mild deterioration. The Areas with more impairment were the area of abstraction (calcuIation) $46.2 \%$ had severe deterioration in the area of short-term memory $47.4 \%$ severe cognitive impairment was found and in the language area was $11.5 \%$

Conclusions: Evaluate and detect cognitive impairment will allow awareness to the family, look for models of care and attention to strengthen the welfare of the elderly

Keywords: cognitive state, elderly, cognitive déficit.

\section{4}




\section{INTRODUCCIÓN}

A ctualmente en el mundo se ha visto un incremento en la población mayor de 60 años de edad. Según datos del INEGl en México se espera que para el año 2050 habrá un 21.5\% de personas en este grupo de edad'.El envejecimiento de la población aumenta la prevalencia de enfermedades crónicas relacionadas con la edad y el incremento de enfermedades neurodegenerativas. ${ }^{2}$

Hoy en día se entien de la vejez como proceso evolutivo, gradual, natural e irreversible, este comienza desde el momento de nacer y termina en la muerte. Es la acción del tiempo sobre los seres vivos que provoca cambios bioquímicos, fisiológicos, morfológicos, sociales y funcionales. ${ }^{3}$

La vejez está relacionada con la reducción de las capacidades funcionales y las alteraciones del estado cognitivo. Teniendo en consideración que el estado cognitivo es un proceso de bio - psicosocial por el cual se recibe la información, se procesa y se entrega un tipo de respuesta. El deterioro cognitivo debe considerarse como la presencia de cualquier alteración en las funciones intelectuales, los cambios del estado cognitivo pueden vincularse con factores como enfermedades degenerativas, aislamiento social y el envejecimiento. ${ }^{4}$

El deterioro cognitivo hace que el adulto mayor sea más dependiente, limita su capacidad de autocuidado y genera cuidados a largo plazo. Cuando existe una alteración del estado cognitivo hay una disminución de la capacidad de funcionamiento y en el dominio de las actividades de la vida diaria4. Es por esto que el estado cognitivo debe ser evaluado para detectar cambios del mismo y de ser necesario hacer un diagnóstico. ${ }^{5}$

El alarmante crecimiento de la población mayor de 60 años provoca el surgimiento de nuevas demandas y consideraciones para el cuidado integral del adulto mayor, conocer el estado cognitivo de la población permite tener un marco de referencia para la creación de modelos de cuidado para este grupo. Teniendo en cuenta lo expuesto anteriormente, se realizó una investigación del estado cognitivo en adultos mayores de 60 años en una casa de rehabilitación y reposo en la ciudad de México haciendo uso del instrumento Mini Examen Mental de Folstein (Mini-Mental) que establece un diagnóstico de las funciones cognitivas. Se tiene como objetivo valorar el estado cognitivo del adulto mayor e identificar las capacidades cognitivas más afectadas según su género, edad y escolaridad.

\section{MATERIAL Y MÉTODOS}

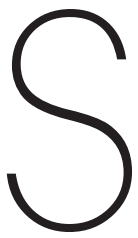
e realizó un estudio de tipo descriptivo transversal no probabilístico con enfoque cuantitativo, en una casa de rehabilitación y reposo ubicada en la Colonia San Jerónimo en México D.F. La población con la que se trabajó fue de 78 adultos mayores de 60 años, donde 41 eran mujeres y 37 hombres. Para la selección de los participantes se tuvieron criterios de inclusión: personas que residian en el asilo, que fueran mayores de 60 años y que dieron su consentimiento para ser incluidas en el estudio. Dentro de los criterios de exclusión se tuvo en cuanta: personas diagnosticadas con demencia, enfermedades neurodegenerativas y personas que no aceptaron participar en el estudio. La recolección de datos consistió en la realización de una valoración del estado cognitivo con ayuda del Mini Examen Mental de Folestein (Mini-Mental). El cual es una prueba de diagnóstico de tamizaje (diagnóstico probable). Que tiene como objetivo establecer un diagnóstico presuntivo de las funciones cognitivas.

Para este estudio se tuvieron en cuenta cuatro variables: el estado cognitivo, género, edad y escolaridad. La aplicación del Mini-Mental fue en la casa de rehabilitación y reposo, se procuró que fuera en un lugar tranquilo, el tiempo de aplicación fue

Vol.74. No. 2 mayo-agosto 2075 w. W. 
de 15 minutos. Para el análisisse diseñó una base de datos a partir del instrumento en el paquete estadístico SPSS versión 21 en el cual se realizó el análisis de datos con estadística descriptiva y para la creación de gráficas se utilizó Microsoft Excel 2010.

Para realizar el estudio se tuvieron en cuenta los lineamientos de la Ley General de Salud en materia de investigación, se solicitó a todas personas que participaron el estudio el consentimiento informado de manera verbal explicando en qué consistía y su importancia, también se garantizó el anonimato de cada persona.

\section{RESULTADOS}

\section{CARACTERÍSTICAS DE LA POBLACIÓN}

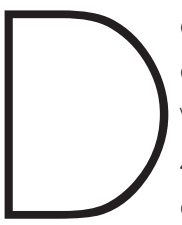
e las 78 personas que fueron incluidas en el estudio, el 47.4\% (37) fueron hombres y el 52.6\% (41) mujeres. Se encontró que 42.3\% (33) estaba en el grupo de edad de 60 a 69 años, el 30.8\%(24) en el grupo de 70 a 79 años de edad, el 21.8\%(17) en el grupo de 80 a 89 años, el 5.1\%(4) en el grupo de 90 a 99 años. No obstante se observó una diferencia entre hombres y mujeres como se aprecia en la Gráfica. 1, en la que se puede ver como el 40.5\%(15) de los hombres se encuentran en el grupo de 60 a 69 años, el 35.1\%(13) en el grupo de 70 a 79 años, el 21.6\% (8) en el grupo de 80 a 89 años, y el 2.8\%(1) en el grupo de 90 a 99 años. Mientras que en el grupo de mujeres el 43.9\%(18) se en encontró en el grupo de 60 a 69 años, el 26.8\%(11) en el grupo de 70 a 79 años, el 21.6\%(9) en el grupo de 80 a 89 años y el 7.4\%(3) en el grupo de 90 a 99 años.

Se encontró que el $5.1 \%$ (4) de los participantes no tuvo ninguna preparación académica, el 21.8\%(17) cursó primaria, el 21.8\%(17) curso secundaria, el 17.9\%(14) cursó preparatoria, el 32.1\%(25) obtuvo un título universitario donde se observó una tendencia en licenciaturas del área físico-matemáticas e ingenierías, así como del área de ciencias sociales, y el 1.3\%(1) curso un posgrado. Pero se observó que en hombres el 2.7\%(1) no cuenta con ninguna preparación académica, el 16.2\%(6) cursó la primaria, el 18.9\%(7) concluyó la secundaria, el 18.9\%(7) estudio la preparatoria, el 40.5\%(15) la licenciatura y el 2.7\%(1) concluyo un posgrado. Mientras que en mujeres se pudo ver que el 7.3\% no cuenta con ningún tipo de preparación académica, el 26.8\% estudió hasta la primaria, el 24.4\% concluyó la secundaria, el 17.1\% estudió la preparatoria y el 24.4\% obtuvo un grado universitario. Como lo vemos en la Gráfica. 2.

De los adultos mayores de 60 años se observó que el 23.1\%(18) presenta deterioro cognitivo severo, el 44.9\%(35) con deterioro cognitivo leve y el 32.1\%(25) no muestra evidencia de deterioro cognitivo. Sin embargo el 21.6\%(8) de hombre presentó deterioro severo, el 45.9\%(17) de deterioro leve, y un 32.4\% (12) fue normal. En mujeres el 24.4\%(10) en deterioro severo, el 43.9\% (18) mostró deterioro leve, y un 31.7\%(13) fue normal. Esto se puede ver en la Gráfica.3.

En el área de orientación temporal se observó que el 16.7\%(13) manifestó deterioro severo, el 38.5\%(30) deterioro leve y 44.9\%(35) no manifestó deterioro en esta área. En el área de orientación espacial se encontró que el 10.3\%(8), presenta deterioro severo, 21.8\%(17) deterioro leve y 67.9\%(53) no mostró deterioro cognitivo, estos resultados fueron similares en ambos géneros.

El resultado obtenido en el área de abstracción (cálculo) fue de 46.2\% (36) con deterioro severo, 23.1\% (18) deterioro leve y 30.8\% (24) no presentaron deterioro. Sin embargo se observó que la población masculina presentó deterioro severo en el 35.1\%(13), de igual forma se observó que el 35.1\%(13) manifestó deterioro leve y un 29.7\%(11) no presentó deterioro. $Y$ el 56.1\%(23) de la población de femenina mostró deterioro severo, y el 12.27\%(5) deterioro leve y un 31.7\%(13) no presentó deterioro como lo podemos observar en la Gráfica.4.

\section{6}

IMNVWhy vol.74. No. 2 mayo-agosto 2015 
En el área de memoria a corto plazo se encontró que los adultos mayores el 47.4\% (37) tuvo deterioro severo, el 25.6\%(20) deterioro leve y 26.9\%(21) no presentaron deterioro. Mas sin en cambio se observó que la población masculina presentó deterioro severo en el 54\%(20) de la población, así mismo notamos que sólo un 18.9\%(7) manifestó deterioro leve y un 27.0\%(10) no mostro signos de deterioro. En la población femenina el 41.5\%(17) presentó deterioro severo, un 31.7\%(13) presentó deterioro leve y un 26.8\%(11) no se observó deterioro como lo vemos en la Gráfica.5.

En la evaluación se halló que en el uso de lenguaje en adultos mayores el 17.5\%(9) manifestaron deterioro severo, el 34.6\% (27) deterioro leve y 56.8\% (42) no presentaron deterioro. De la misma forma se observó que en hombres se encontró deterioro severo en el 13.5\%(5) de la población, el 35.1\%(13) de evaluó con deterioro leve y un 51.4\%(19) no presenta deterioro. Mas sin en cambio la población femenina presentó el 9.8\% (4) deterioro severo, el $34.1 \%(14)$ deterioro leve y un 56.1\%(23) no manifestó deterioro como se puede apreciar en la Gráfica.b.

La evaluación del área de comprensión de instrucciones mostró que el 29.5\% presentó deterioro severo, el $12.8 \%$ deterioro leve y el 57.7\% no presentaron deterioro.En el área de Percepción viso -espacial se encontró que el 44.9\% no manifestó datos de deterioro, mientras el 55.1\% no mostró deterioro, estos valores fueron parecidos entre hombres y mujeres.

\section{DISCUSIÓN}

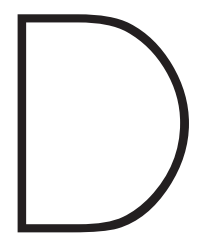

ebido a la selección de muestra no probabilística los resultados solo pueden ser aplicados a la población de estudio. A pesar de no encontrar una diferencia significativa vemos que existe un ligero predomino en la presencia deterioro cognitivo en mujeres, algunos estudios establecen una relación entre el género femenino y el riesgo de presentar deterioro cognitivo como los menciona
Mías C.D. en su estudiob, los resultados encontrados en esta investigación apuntan a una posible relación entre ambos. Aunque los resultados también sugieren que la diferencia podría deberse a que en la población femenina predominan personas de mayor edad, ya que con el incremento de la edad existe un aumenta en la amenaza de padecer algún tipo de deterioro en el estado cognitivo como se comprueba en diversos artículos ${ }^{7-8 .}$

Diferentes estudios afirman que un alto grado de educación formal disminuye el riesgo de padecer una afectación en las funciones cognitivas ${ }^{9-10}$ los resultados de esta investigación no coinciden con lo mencionado por Samper', en este estudio se encontró que la población masculina que cuenta en su mayoría con estudios de nivel superior y medio superior apenas muestran una diferencia en el estado cognitivo en comparación con las mujeres, las cuales en su mayoría solo cuanta con educación básica. Solo en el área de abstracción (cálculo) coinciden con lo encontrado por Samper donde vemos que la disminución de la escolaridad puede ser un elemento que afecte de manera negativa el deterioro. A pesar que ambos géneros mostraron un alto porcentaje de de terioro que coincide con los resultados obtenidos por González" que observó un alto porcentaje de deterioro en el área de atención y cálculo, en este estudio se aprecia que en la población femenina poco más de la mitad mostró un deterioro severo en esta área, lo cual podría deberse a que el nivel de escolaridad es menor al de la población masculina.

Así mismo se observó que en el área de memoria a corto plazo existe un alto porcentaje de deterioro en ambos géneros con un tenue predominio en la presencia de deterioro severo en hombres, lo que se asemeja con lo encontrado en la literatura que explica que el envejecimiento, los cambios fisiológicosen el cerebro así como la disminución de neuronas y dendritas se relaciona con el daño a la memoria ${ }^{12}$. 
En el área de uso de lenguaje encontramos que más de la mitad de los participantes manifestaron un deterioro en esta área, lo cual podría deberse al enlentecimiento del procesamiento de información y disminución de la atención ${ }^{12}$, sin embargo hay una diferencia entre ambos géneros donde vemos un mayor porcentaje de deterioro en hombres a comparación con las mujeres, esto podría deberse al hecho que la mujer mantiene un nivel mayor de interacciones con la familia e hijos, esto provoca un mayor número de estímulos cognitivo-afectivo que se cree que disminuye el riesgo de padecer deterioro en el estado cognitivo?

Las alteraciones del estado cognitivo constituyen un factor que influye de manera negativa en el mantenimiento de la autonomía ${ }^{5}$ y el autocuidado, lo que genera que el adulto se vuelva dependiente de los cuidadores primarios y personal de salud, el deterioro cognitivo eleva las demandas de cuidados al no ser capaz de realizarlos por sí mismo.El personal de enfermería al ser un profesional que mantiene una estrecha relación con el adulto mayor debe tener conocimiento del estado cognitivo para generar planes de cuidados que se ajusten a las necesidades individuales de cada caso.

Los profesionales de enfermería deben ser promotores de la vigilancia y el diagnóstico de los cambios en el estado cognitivo en adultos mayores para buscar estrategias conjuntas con los cuidadores para disminuir en lo posible el efecto negativo en la calidad de vida de adulto mayor ${ }^{13}$. El envejecimiento de la población genera que el deterioro cognitivo sea más frecuente en las personas de edad avanzada, lo cual sugiere que los modelos de atención en los diferentes niveles se preparen para cumplir con las demandas que surgen con el aumento de dependencia y cuidados que requiere el adulto mayor, por lo tanto es necesario la creación de nuevas políticas encaminadas hacia modelos que ofrezcan una atención sostenible y de calidad.

\section{CONCLUSIÓN}

e puede concluir que en la población
estudiada hay un mayor deterioro en las
áreas de abstracción (cálculo), memoria
a corto plazo y uso del lenguaje.
El estudio mostró que en la población masculina las áreas con mayor deterioro severo son: lenguaje, orientación espacial, memoria corto plazo y percepción viso-espacial. Mientras que en la población femenina las áreas con mayor deterioro severo son: Comprensión de instrucciones, orientación temporal y la abstracción (cálculo).

El deterioro cognitivo es uno de los padecimientos más importantes que presentan con mayor prevalencia en adultos mayores. Sin embargo no solo es un problema que afecta a quien lo padece, sino también afecta al cuidador primario y a la familia de este. Por esta razón es necesario que entiendan el porqué de cierto comportamiento de las personas de edad avanzada y sobre las características de las alteraciones cognitivas, evaluar y detectar a tiempo el deterioro cognitivo, así como prevenirlo y tratarlo. De esta manera los profesionales de enfermería juegan un papel importante en la capacitación y orientación de los cuidadores primarios y la familia. De igual forma es necesario buscar o formar redes de apoyo que nos permita disminuir el riesgo de caer en una situación que ponga en riesgo la vida de la persona con deterioro cognitivo como depresión o accidentes en el hogar. De esta manera se puede contribuir con cuidado integrales para mejorar la calidad de vida y se reforzará la seguridad en su entorno.

\section{Agradecimientos:}

Brindamos nuestro más sincero agradecimiento a todas las personas que hicieron posible la realización de esta investigación en especial a Sánchez Hernández Guadalupe Irene, López Franco Laura Cristina, Peralta Carbajal Silvia y Mata Vilchis Wendy por su valiosa aportación y colaboración para la elaboración de esta investigación.

\section{8}

भMN MWhar vol.14. No.2 mayo-agosto 2015 


\section{REFERENCIAS BIBLIOGRÁFICAS}

1. Instituto Nacional de Estadística y Geografía. Estadísticas a propósito del día internacional de las personas de edad (internet). 2013. Disponible: http://www.inegi.org.mx/inegi/contenidos/espanol/prensa/Contenidos/estadisticas/2013/adultos0.pdf.

2. Monteagudo $M$, Gómez $N$, Martín $m$, Jiménez L, Mc Cook E, Evaluación del estado cognitivo de los adultos mayores de 60 años; en el área de salud del policlínico docente plaza de la revolución. Revista Cubana de Medicina. 2009: 48: 59-70.

3. Rodríguez K. Vejez y envejecimiento. 1" ed. Ed Universidad del Rosario, Colombia; 2011: 15-16.

4. Varela L, Chávez H, Méndez F, Características del estado cognitivo en el adulto mayor hospitalizado a nivel nacional. Rev. Soc. Per. Med. 2005: 17: 37-39.

5. Álvarez J, Sicilia M, Deterioro cognitivo y autonomía personal básica en personas mayores. Anales de psicología (PDFInternet). 2007 (consulta 20-11-2014) 23(2):272-278 Disponible en: http://www. readalyc.org/articulo.oa?id=167232214.

6. Mías C, Sassi M, Masih M, Querejeta A, Krawchik R, Deterioro cognitivo leve: estudio de prevalencia y factores sociodemográficos en la ciudad de Córdoba, Argentina (PDF Internet) 2007 (consulta 19-01-2015) 44(12): 733-738 Disponible en: www.neurologia.com/ pdf/web/4412/x120733.pdf.

7. Cruz M, Deterioro cognitivo en la población mayor de 65 años que reside en el área metropolitana de Monterrey, México. Medicina Universitaria 2008: 10 (40): 154-158.
8. De león R, Milián F, Camacho N, Arévalo R, Escartin M, Factores de riesgo para deterioro cognitivo y funcional en el adulto mayor Rev. Med. Inst. Mex. Seguro. Soc. (PDF Internet) 2009(consulta 18-012015) 47(3):277-284 Disponible en: http://www.medigraphic.com/ pdfs/imss/im-2009/im093h.pdf.

9. Samper J, Llibre J, Sánchez G, Pérez C, Morales E, Sosa S, et. al. Edad y escolaridad en sujetos con deterioro cognitivo leve. Rev. Cub. Med. Mil, (PDF Internet) 2011 (consulta 19-01-2015) 40 (3) Disponible en:http://scielo.sld.cu/scielo.php?script=sci_arttext\&pid=S0138-6557201700030000 1

10. Ávila $M$, Vázquez E, Gutiérrez $M$, Deterioro cognitivo en ele adulto mayor. Ciencias Holgin (PDF Internet) 2007 (consulta 19-0120015) 13 (4): 1-11 Disponible en: http://www.redalyc.org/articulo. oa? id $=187517998004$.

11. González J. Gómez N, González J.L, Marín M Deterioro cognitivo en la población mayor de 65 años de dos consultorios del policlínico fLa Rampa. Rev. Cubana Med Gen Integr (Internet) 2006 (consulta 19-01-2014) 22 (3) Disponible en: http://bvs.sld.cu/revistas/mgi/ vol22_3_06/mgi05306.htm.

12. Salech F, Jara R, Michea L, Cambios Fisiológicos asociados al envejecimiento Rev. Med. Clin. Condes 2012: 23 (1): 19-29.

13. Hernández E, Gonzales A, Moreno Y, Deterioro cognitivo y calidad de vida en ancianos de una clínica de medicina familiar de la ciudad de México Archivos en Medicina Familiar (PDF Internet) 2007 (consulta 19- 01-2015) 10(4): 127-132 Disponible en: http:// www. Realdyc. org/articulo. oa?id=50719094002 


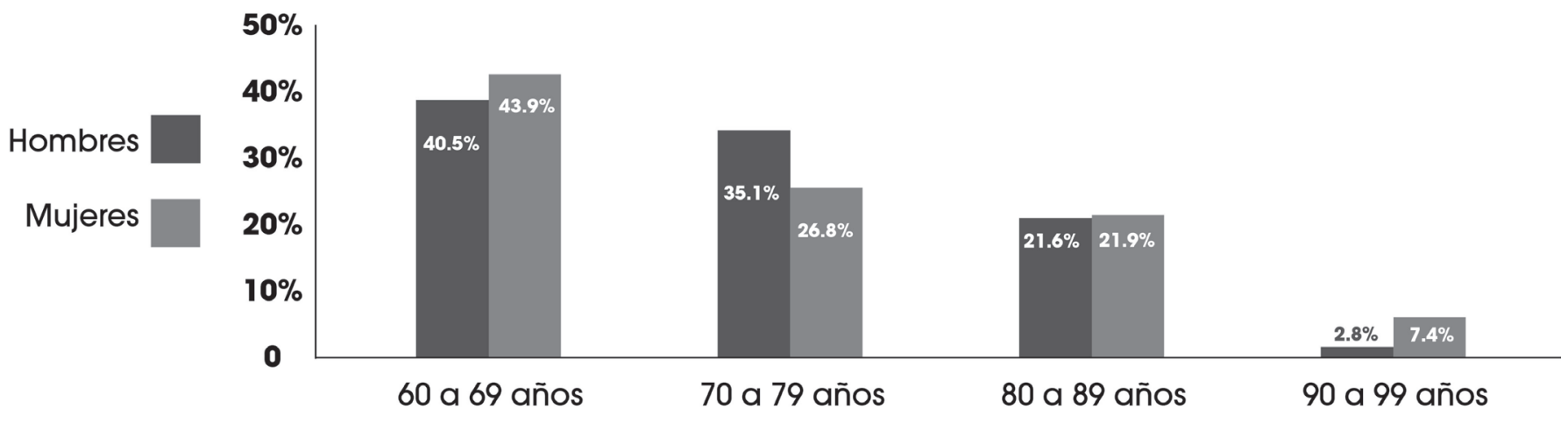

$\mathrm{N}: 78$

Fuente directa: Evaluación de Mini Examen Mental de folstein

Gráfica 2: Distribución de nivel de escolaridad por género en adultos mayores de 60 años



Gráfica 3: Distribución del estado cognitivo en adultos mayores de 60 años por género

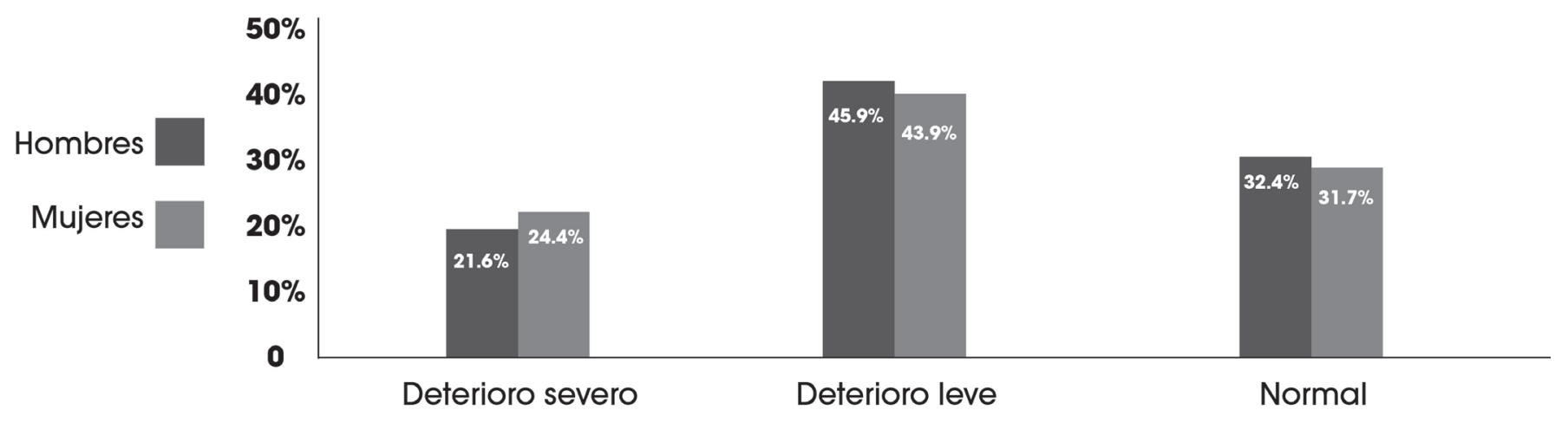




\section{Gráfica 4: Distribución de abstracción (cálculo) en adultos mayores de 60 años por género}

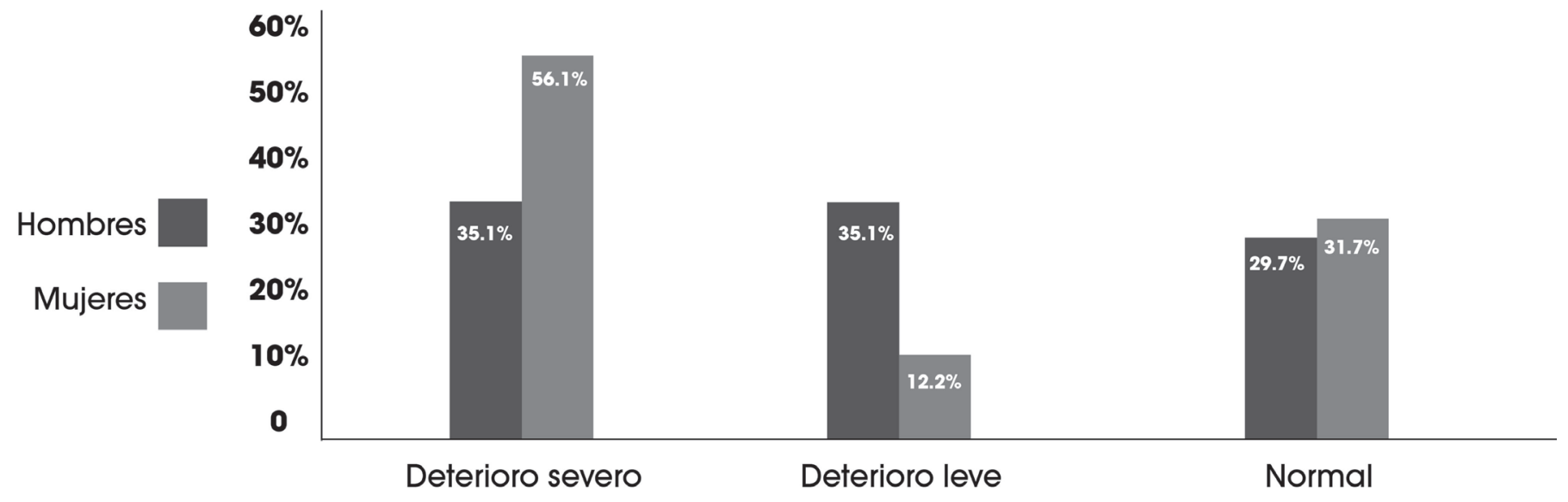

Gráfica 5: Distribución de memoria a corto plazo en adultos mayores de 60 años por género

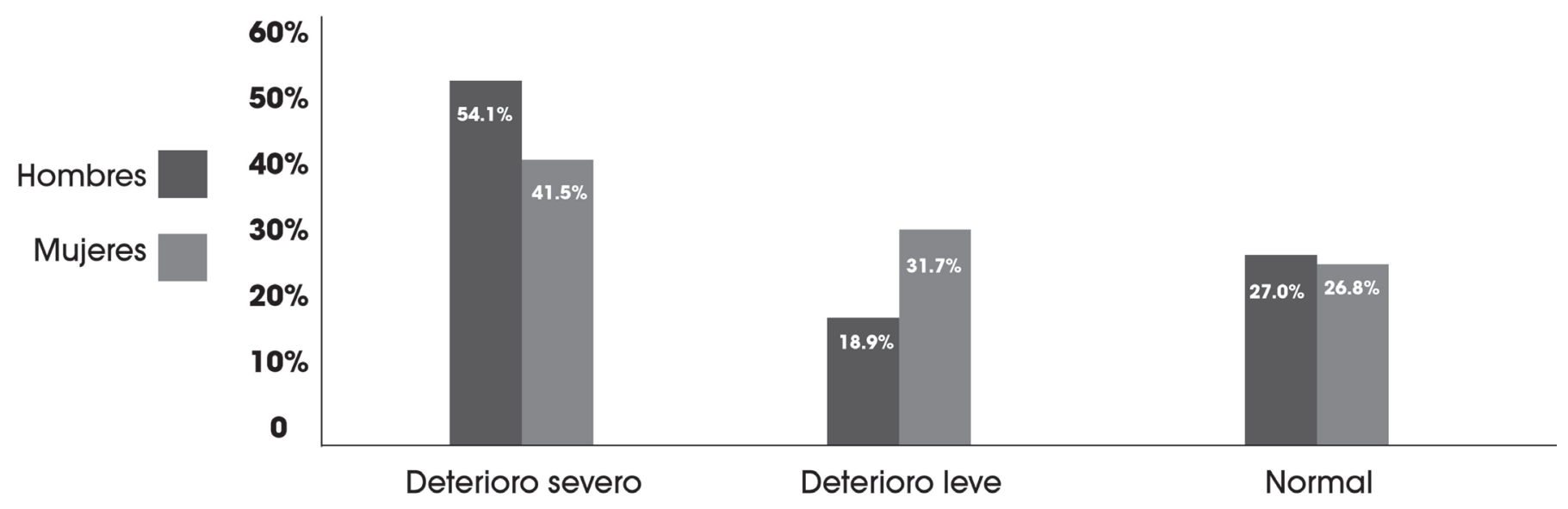

Gráfica 6: Distribución del uso de lenguaje en adultos mayores de 60 años por género

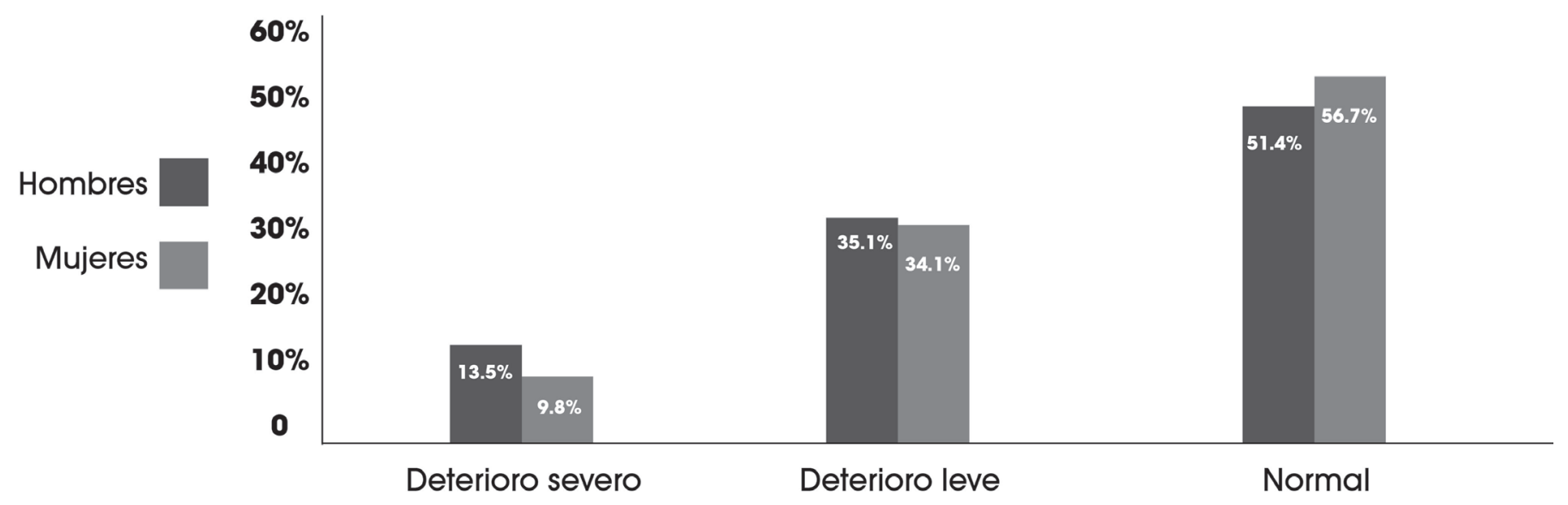

